\section{PANCREAS PATHOLOGY OF HUMAN ARX-NULL MUTATION: CONSIDERATION OF PANCREAS ENDOCRINE CELLS DIFFERENTIATION}

\section{Itoh}

National Center of Neurology and Psychiatry, Kodaira, Japan

Aristaless-related homeobox gene $(A R X)$ mutation leads to several neurological disorders including $X$-linked lissencephaly with abnormal genitalia (XLAG), West syndrome and Partington syndrome, with XLAG being the most severe form. Although some of the brain pathology of XLAG has already been described, the crucial extra-brain symptoms are severe growth retardation, transient hyperglycemia and intractable diarrhea. Since ARX expresses in the islets of Langerhans during the embryonic stage, these visceral phenotypes may be related to a loss of ARX function, which develops endocrine cells in the pancreas. We investigated the abnormal pancreatic development of XLAG patients with $A R X$ null mutation. We performed immunohistochemistry of XLAG pancreases, using the antibodies against glucagon, insulin, somatostatin, pancreatic polypeptide, ghrelin, Brn4, Nkx2.2, Mash1, Pdx1, amylase and pancreatic lipase. As the results, the glucagon- and pancreatic polypeptide-producing cells were found to be completely deficient in the islets of Langerhans. We also discovered marked interstitial fibrosis, small exocrine cells with loss of amylase-producing cells and an enlargement of the central lumen of the glandular acini. These pathological findings indicate that ARX contributes not only to endocrine development, but also to exocrine development of the human pancreas, and its deficiency may lead to the severe phenotypes of XLAG patients.

\section{FREE THYROXINE LEVELS IN THE INITIAL FOUR WEEKS OF LIFE IN EXTREME PRETERM INFANTS AFFECT BRAIN GROWTH}

S.M. Ng${ }^{1}$, M.A. Turner ${ }^{1}$, M. Didi², C. Gamble ${ }^{3}$, S. Victor ${ }^{4}$, D. Manning ${ }^{5}$, P. Settle ${ }^{6}$, R. Gupta ${ }^{7}$, A.M. Weindling 1

${ }^{1}$ School of Reproductive and Developmental Medicine, University of Liverpool, ${ }^{2}$ Department of Endocrinology, Alder Hey Hospital, ${ }^{3}$ Centre for Medical Statistics and Health Evaluation, University of Liverpool, Liverpool, ${ }^{4}$ School of Clinical and Laboratory Sciences, University of Manchester, Manchester, ${ }^{5}$ Neonatal Unit, Wirral

University Teaching Hospital, Wirral, ${ }^{6}$ Neonatal Unit, Salford Royal NHS Foundation Trust, Salford,

${ }^{7}$ Neonatal Unit, Royal Preston Hospital, Preston, UK

Background: Low blood thyroid hormone concentrations in the first few weeks of life in extremely preterm infants may be linked with poor neurodevelopment. We conducted a multi-centre randomised controlled trial of thyroid hormone supplementation in babies under 28 weeks' gestation.

Methods: 78 infants received levothyroxine supplementation and 75 received placebo. There was no difference in overall outcome. A post hoc subgroup analysis was undertaken. FreeT4 (FT4) plasma levels were measured longitudinally during the initial 4 weeks after birth. Two subgroups with average FT4 values in the lowest and highest quartiles were created for each trial arm. Univariate and multivariable analyses comparing the lowest and highest quartiles of FT4 within each trial arm were undertaken. Width of subarachnoid space at 36 weeks corrected for postmenstrual age was used as a measure of brain volume.

Results: Among placebo infants, univariate analyses showed that the lowest quartile of FT4 was associated with larger subarachnoid space (smaller brain volume) $[95 \% \mathrm{Cl}=0.05(0.002,0.09)]$. These infants also had higher mortality, lower gestational age and lower birth weight compared with those in the highest quartile. In the thyroxine group, there was no significant difference in subarachnoid space between the lower and higher quartiles.

Conclusion: Van Wassanaer (2002) reported that low FT4 levels in the first 4 weeks of birth were associated with worse neurodevelopmental 
outcome at 5 years in babies born < 30 weeks. Our findings extend their work by suggesting that longitudinal FT4 levels are an independent factor affecting brain growth in babies born $<28$ weeks.

\section{2}

\section{LONGITUDINAL CHANGE OF LIVER FAT IN OBESE CHILDREN AFTER A 12-MONTHS NUTRITIONAL INTERVENTION}

\author{
E. Verduci ${ }^{1}$, G. Radaelli ${ }^{1}$, S. Scaglioni ${ }^{1}$, \\ G. Banderali ${ }^{1}$, M. Salvioni ${ }^{1}$, C. Pozzato ${ }^{2}$,
} M. Giovannini ${ }^{1}$

${ }^{1}$ Department of Pediatrics, ${ }^{2}$ Department of Radiology, San Paolo Hospital, University of Milan, Milan, Italy

Objective: To evaluate whether an one-year nutritional-behaviour intervention based on normocaloric balanced diet and physical exercise may reduce the liver fat in obese children.

Methods: Twenty-six obese children (11 males and 15 females), aged 6-14 years, underwent anthropometic, nutritional, metabolic and liver magnetic resonance imaging (MRI) examinations at baseline and after an one-year nutritionalbehaviour intervention. Anthropometry included weight, height, waist and hip circumference and total upper arm area. Body mass index (BMI) z-scores were calculated. Biochemistry included serum aminotransferases, lipid profile, glucose and insulin. Liver steatosis was judged as hepatic fat fraction (FF) by MRI was $\geq 9 \%$.

Results: Prevalence of steatosis was $34.6 \%$ at baseline and declined to $7.7 \%$ after intervention $(\mathrm{P}<0.0001)$. Mean $(95 \% \mathrm{Cl})$ reduction of liver $\mathrm{FF}$ was $8.0(4.0-12.0) \%$. In $77.8 \%$ of children with liver steatosis at baseline the FF declined lower than $9 \%$ at the end of intervention, going from a mean [SD] of 18.7 [9.1] \% to 1.3 [4.1] \%, $(\mathrm{P}<0.0001)$. At the end of the intervention children showed a mean reduction in BMI z-score of $0.26(0.11$ $0.41)$, and waist circumference of 1.46 (0.34-2.60) $\mathrm{cm}$. Triglycerides, total cholesterol, ApoA1, ApoB, ApoA1/ApoB ratio and gamma-GT plasma values in plasma decreased at the end of intervention $(\mathrm{P}<$ 0.05).

Conclusions: The results suggest that in obese children nutritional-behaviour interventions may reduce the liver fat.

\section{3}

\section{VALIDATION OF BIOELECTRICAL IMPEDANCE ANALYSIS WITH A THREE-COMPONENT MODEL OF BODY COMPOSITION IN 4-7 YEAR OLD CHILDREN}

\author{
M. de Beer ${ }^{1}$, T. Timmers ${ }^{1}$, P.J.M. Weijs ${ }^{2}$, \\ R.J.B.J. Gemke ${ }^{1}$ \\ ${ }^{1}$ Pediatrics, VU University Medical Centre, \\ ${ }^{2}$ Department of Nutrition and Dietetics, Internal \\ Medicine, VU University Medical Center, \\ Amsterdam, The Netherlands
}

Field methods to assess body composition in young children are scarce. The aim of this study was to validate bioelectrical impedance analysis (BIA) with the three-component (3C) model of body composition in primary school children.

We examined fifty-six 4-7 year old children, 36\% boys. Total body water estimated by BIA was compared with deuterium dilution. An established equation was recalibrated using a validation sample of 36 children which was subsequently crossvalidated in an independent sample of 20 children. Furthermore, commonly used gender-specific hydration constants for the conversion of total body water into fat free mass were compared to those calculated with the $3 \mathrm{C}$ model.

Compared to deuterium dilution, BIA for school age children underestimated total body water by $0.51 \mathrm{~kg}(p=0.002)$ and this varied across the range of TBW $(r=0.41 ; p=0.002)$. Recalibration of the equation yielded: Total body water $=0.439$ (Height $2 /$ Resistance $)+0.027 \mathrm{Wt}+4.014\left(R^{2}=0.74\right.$, SEE $=0.96 \mathrm{~kg}$ ). Application of this equation in the independent sample of 20 children showed a nonsignificant bias in total body water (mean bias $-0,40$ $\mathrm{kg}, \mathrm{p}=0.223)$. This bias was not variable $(r=-0.11$; $p=0.648$ ). Hydration constants calculated with the $3 \mathrm{C}$ model were comparable to recently proposed hydration constants.

In conclusion, these data indicate that BIA underestimates total body water in 4-7 year old children, when using the Kushner equation for school age children. This underestimation is nonsystematic. Recalibration and cross-validation enables more robust assessment of body composition for routine survey and clinical use in (pre)school aged children. 